\title{
Robust prediction of residual velocities and ballistic limits of projectiles for impact on thin aluminium plates
}

\author{
M. Raguraman \& A. Deb \\ Centre for Product Design and Manufacturing, \\ Indian Institute of Science, Bangalore, India
}

\begin{abstract}
This paper deals with the simulation-driven study of the impact of hardened steel projectiles on thin aluminium target plates using explicit finite element analysis as implemented in LS-DYNA. The evaluation of finite element modelling includes a comprehensive mesh convergence study using shell elements for representing target plates and the solid element-based representation of ogivalnosed projectiles. A user-friendly automatic contact detection algorithm is used for capturing interaction between the projectile and the target plate. It is shown that the proper choice of mesh density and strain rate-dependent material properties is crucial as these parameters significantly affect the computed residual velocity. The efficacy of correlation with experimental data is adjudged in terms of a 'correlation index' defined in the present study for which values close to unity are desirable. By simulating laboratory impact tests on thin aluminium plates carried out by earlier investigators, extremely good prediction of experimental ballistic limits has been observed with correlation indices approaching unity. Additional simulation-based parametric studies have been carried out and results consistent with test data have been obtained. The simulation procedures followed in the present study can be applied with confidence in designing thin aluminium armour plates for protection against low calibre projectiles.
\end{abstract}

Keywords: residual velocity, ballistic limit, projectile, aluminium plate, finite element modelling, LS-DYNA. 


\section{Introduction}

The behaviours of armour plates under projectile impact has been studied experimentally [1-6], using analytical/semi-empirical formulations [2, 7] and numerically [8-15]. Amongst these three approaches, the numerical analysis procedure consisting of nonlinear finite element-based analysis appears to be the most versatile tool for predicting ballistic limits of projectiles for impact on armour plates. The primary objective of the numerical studies [8-15] reported by different investigators was to show that analysis results can correlate against experimental results including failure. Most of the finite element modelling procedures discussed in literature employed plane strain or axisymmetric finite element representation of projectiles and target plates. However, a limitation of this approach lies in the difficulty of assessing normal/oblique impacts on targets of complex shapes; also an excessively refined mesh of the target is required in such 2D representations. In the present study, plates are represented with shell elements and projectiles with solid elements. Published data on stress-atrain behaviours of an aluminium alloy (Al 6061 T6) have been used to generate the strain rate-dependence of yield and failure stresses of the target plate material (Al $1100 \mathrm{H14}$ ) studied here. This procedure of material modelling with a Von Misestype yield criterion and isotropic strain hardening can be appealing to design engineers for its inherent simplicity. While material modelling is a key consideration in analyzing the mechanics of projectile and target interaction, other parameters such as mesh density cannot be ignored. In order to establish the required modelling criterion in terms of element size, a convergence study of residual velocity has been carried out by varying mesh density in the impact zone. Additionally, the effect of using quasi-static properties only of plate material without considering strain rate sensitivity is shown to yield residual velocities which are significantly higher than corresponding test velocities. Using the preferred material and meshing criteria arrived at here, extremely good prediction of test residual velocities and ballistic limits given in [2] for thin aluminium plates has been obtained. The sumulation-based pattern of failure with localized bulging and jagged edge of perforation is similar to the perforation with petalling seen in tests.

\section{Finite element modelling}

Finite element models of a thin aluminium target plate and a hardened steel projectile using shell and solid elements respectively are shown in Figure 1. For analysis using LS-DYNA, 4-node Belytschko-Lin-Tsay shell elements based on a co-rotational formulation are used. The plate is circular in shape with a diameter of $255 \mathrm{~mm}$ and is clamped along the edges. Plates of three different depths (thickness) viz. $0.5 \mathrm{~mm}, 1.5 \mathrm{~mm}$ and $2.0 \mathrm{~mm}$ are considered. The projectiles are generally of diameter $10 \mathrm{~mm}$ or $15 \mathrm{~mm}$, and are ogival-nosed. The length to diameter $(\mathrm{l} / \mathrm{d})$ ratio of $10 \mathrm{~mm}$ diameter projectiles was varied from 1 to 4 while studying the effect of projectile mass on ballistic limit in the parametric 
studies carried out. CONTACT_AUTOMATIC_GENERAL interface is defined for capturing the interaction between target plate and projectile.

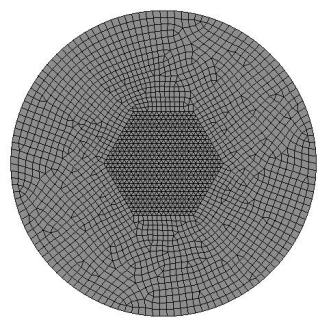

(a)

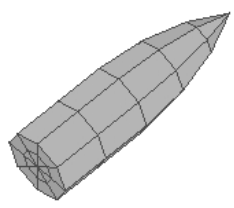

(b)

Figure 1: (a) Plate modelled with shell elements; (b) Projectile modelled with solid elements.

\subsection{Material modelling}

The material model of type 19 in LS-DYNA has been used here for defining the behaviour of target aluminium plates studied experimentally in [2]. In the constitutive model used, yield and tensile strengths can be specified in a tabular manner with respect to effective strain rate. The information furnished in [2] on the target plate material indicates that it is closest to Al $1100 \mathrm{H} 14$ alloy for which quasi-static properties are given in [16]. The strain rate-dependent behaviour of this alloy was not readily available. However, test-based values of yield and ultimate strengths at a number of strain rates are mentioned in [17] for the $\mathrm{Al}$ 6061 T6 alloy. The variations of material properties (i.e. yield and ultimate stresses) for this latter alloy can be represented with a fair degree of fidelity to test data with the help of second order regression polynomials as shown in Figure 2. These regression polynomials can be used for predicting the yield and ultimate strengths of Al 6061 T6 alloy at any strain rate within the ranges given in Figure 2. The strengths of the plate material (Al $1100 \mathrm{H} 14$ alloy) are then obtained by scaling as per equation (1) given below:

$$
\sigma_{\dot{\varepsilon}}^{1100}=\sigma_{\dot{\varepsilon}_{0}}^{1100} \cdot \frac{\sigma_{\dot{\varepsilon}}^{6061}}{\sigma_{\dot{\varepsilon}_{0}}^{6061}}
$$

where, $\sigma_{\dot{\varepsilon}}^{\text {(alloygrade) }}$ is the strength (yield or ultimate) at a given strain rate, $\dot{\varepsilon}\left(\mathrm{s}^{-1}\right)$; and, $\sigma_{\dot{\varepsilon}_{0}}^{(\text {alloy grade })}$ is the corresponding quasi-static strength at a low strain rate of $\dot{\varepsilon}_{0}\left(\mathrm{~s}^{-1}\right)$. 


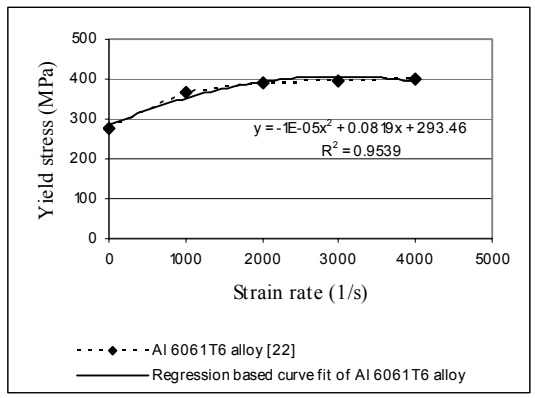

(a)

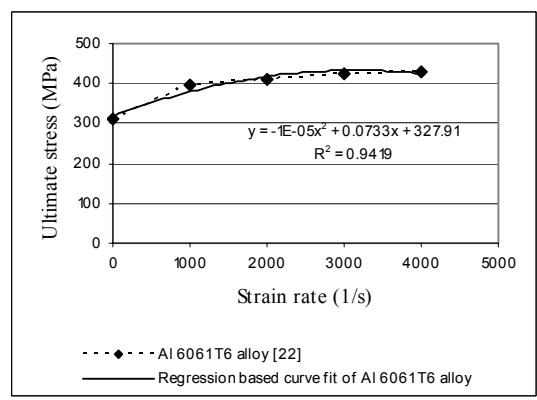

(b)

Figure 2: Regression-based curve-fitting of (a) yield stress, and (b) ultimate stress of Al $6061 \mathrm{~T} 6$ alloy with respect to strain rate.

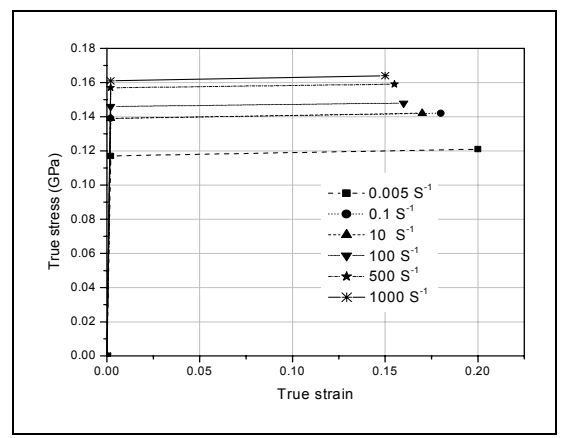

Figure 3: Derived true stress vs. true strain behaviours of Al $1100 \mathrm{H} 14$ alloy at different strain rates.

Following the approach outlined above and assuming a constant tangent modulus obtained from the quasi-static properties of Al $1100 \mathrm{H} 14$ alloy specified in [16], the bi-linear variations of true stress with respect to true strain are obtained as shown in Figure 3 and used in material type 19 as mentioned earlier for representing the behaviour of target plate material in finite element analysis.

The projectile core and sheath are modelled with material type 24 in LSDYNA used for defining piecewise linear elasto-plastic behaviour. The hardness specified in [2] of the steel core of a projectile was found to be comparable to that of AISI A2 tool steel mentioned in [16]. Hence the properties of this hardened steel have been chosen for defining the behaviour of projectile core in current simulations. For all analyses carried out here, the visco-plastic formulation option was turned on for materials of both plate and projectile.

\section{Effect of element size on projectile residual velocity}

The objective of this study is to determine an optimal element size that will yield reliable values of projectile residual velocity. Plates of three different depths 
have been considered and the computed residual velocities are compared (as shown in Figure 4) with corresponding test residual velocities reported in [2]. It is found that residual velocity tends to converge monotonically in all cases considered, and a nearly circular perforation due to removal of elements in the impact zone is obtained. In order to assess the degree of correlation of simulation-based residual velocities with test residual velocities, the following 'Correlation Index' $(C I)$ is defined:

$$
C I=1-\frac{\sqrt{\left(\sum e_{i}^{2}\right)}}{\sum V_{r}}
$$

where, $V_{r}$ is the test residual velocity, $e_{i}$ is the difference between computed and test residual velocities, and the summation is carried out over the number of cases for which a combined index of correlation is sought. It is apparent from (2) that as the degree of correlation increases, $C I$ approaches unity. A $C I$ value of 0.983 has been obtained from Figure 4 by considering all converged residual velocities. It may be concluded from Figure 4 that shell elements of size 2-4 mm may be used for analysing thin aluminium targets with automatic contact detection interface in LS-DYNA.

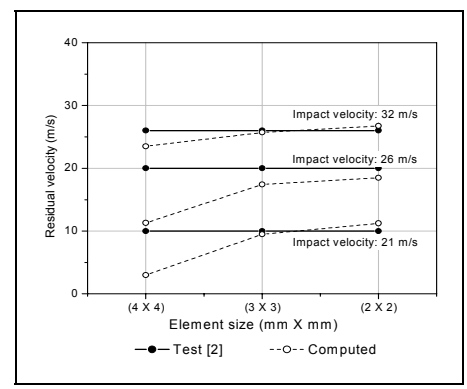

(a)

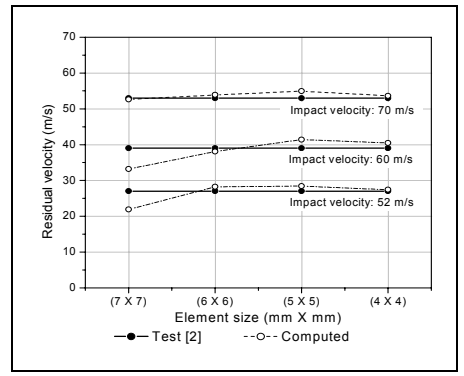

(b)

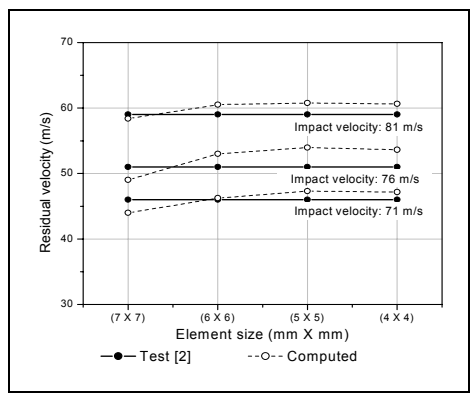

(c)

Figure 4: $\quad$ Mesh convergence study for (a) $0.5 \mathrm{~mm}$, (b) $1.5 \mathrm{~mm}$, and (c) 2.0 $\mathrm{mm}$ thick aluminium target plates. 


\section{Effect of strain rate-dependent material modelling of target on projectile residual velocity}

One of the key aspects of finite element modelling is the adoption of strain ratedependent material behaviour as discussed previously in section 2.1. The effect of strain rates is assessed by repeating the residual velocity convergence study carried out in the previous section for plates of $0.5 \mathrm{~mm}$ and $2 \mathrm{~mm}$ thickness by assigning quasi-static properties only to plate material, and ignoring strain ratesensitive yield and ultimate strengths. It is seen in Figure 5 that ignoring material strain rate sensitivity has a noticeable effect on computed residual velocities that turn out to be significantly higher than the corresponding test-based values. This is a consistent outcome as usage of quasi-static properties at high strain rates is tantamount to using a weaker plate.

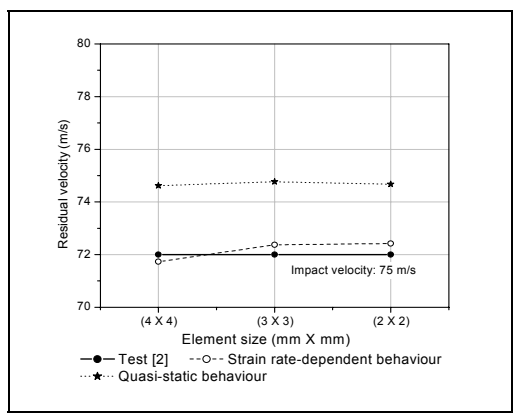

(a)

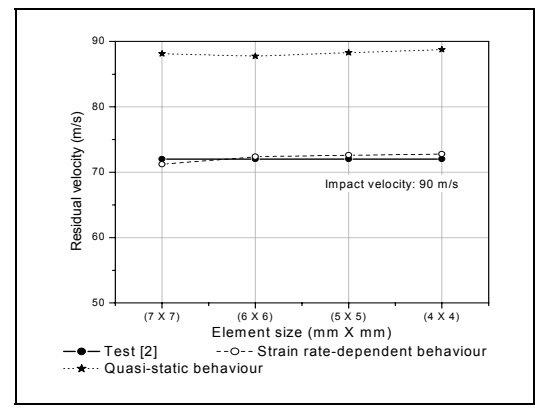

(b)

Figure 5: Effect of ignoring material strain rate sensitivity on computed residual velocity for (a) $0.5 \mathrm{~mm}$, and (b) $2 \mathrm{~mm}$ thick plates

\section{Ballistic limit prediction and other parametric studies}

With the help of the mesh configuration and material modelling procedure that yielded desirable residual velocities in convergence studies reported already, a number of parametric studies are next carried out by varying target plate thickness as well as projectile geometry and mass. The ballistic limits obtained through simulation are compared with corresponding experimental data to establish the power of present modelling approach as an efficient and reliable technique for designing penetration-resistant armours.

\subsection{Definition of ballistic limit}

Ballistic limit is an essential index for evaluating projectile and armour performance and is defined in [5] as "the average of two striking velocities, one of which is the highest velocity giving a partial penetration and the other which is the lowest velocity giving a complete penetration". In the present study, ballistic limit is interpreted as the minimum impact velocity at which zero residual velocity is first encountered. Thus, referring to Figure 6 which depicts 
velocity time histories of projectiles normally impacting a $1.5 \mathrm{~mm}$ thick target plate, $43.5 \mathrm{~m} / \mathrm{s}$ can be considered as the ballistic limit as a slightly higher impact velocity results in a non-zero residual velocity.

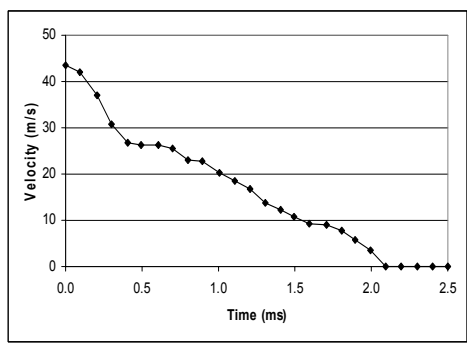

(a)

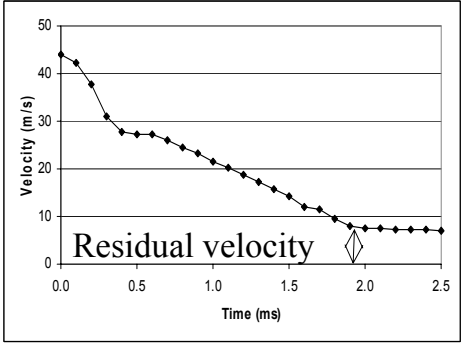

(b)

Figure 6: Computed velocity time histories for $1.5 \mathrm{~mm}$ thick target plates with normal impact speeds of (a) $43.5 \mathrm{~m} / \mathrm{s}$ and (b) $44 \mathrm{~m} / \mathrm{s}$.

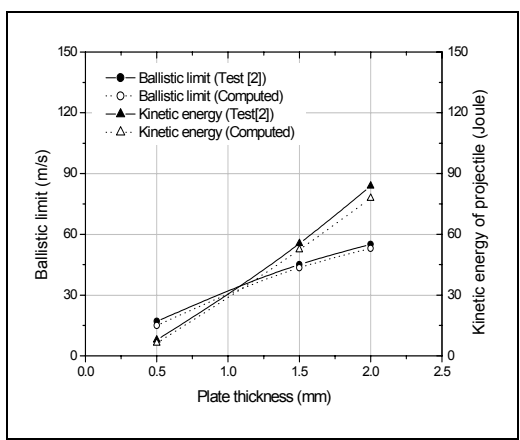

(a)

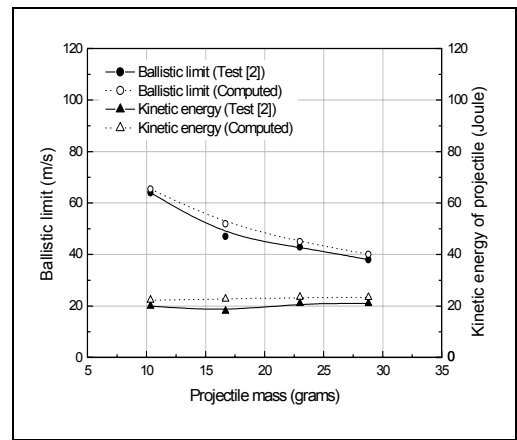

(b)

Figure 7: Variations of ballistic limit and residual kinetic energy of projectile with respect to (a) plate thickness, and (b) projectile mass.

\subsection{Effect of plate thickness on ballistic limit and residual kinetic energy}

Computed ballistic limits and projectile residual kinetic energies are plotted in Figure 7(a) with respect to plate thickness. Both the ballistic limit and the residual kinetic energy of a projectile display rising trends with increasing plate thickness as observed in tests reported in [2]. A CI value of 0.9754 is obtained from Figure 7(a) for all data points considered, which shows that a great degree of correlation exists between experimental data and the computed results obtained here.

\subsection{Effect of projectile mass on ballistic limit and residual kinetic energy}

The effect of changing projectile shank length leads to a change in projectile aspect (1/d) ratio and it's mass. It has been found in [2] and shown in Figure 7(b) that increasing projectile mass in this manner actually decreases its ballistic 
limit; however, projectile residual kinetic energy is found to be relatively insensitive to change in projectile mass when diameter is kept as constant. A comparison is made between the computed ballistic limits and projectile residual kinetic energies for varying projectile mass with the corresponding test-based values in Figure 7(b) and extremely good correlation confirmed by a $C I$ value of 0.9689 is observed.

\subsection{Effect of projectile nose shape on residual velocity}

The investigators in [3] have studied the relative performances of blunt- and hemispherical-nosed projectiles during impact on thin aluminium plates. The numerical simulations were carried out in [3] using 2D elements exploiting axisymmetry and employing the ABAQUS code. Following the present finite element procedure and carrying out analysis with LS-DYNA, a comparison is made in Figure 8 between the computed results and corresponding test data given in [3]. A C I value of 0.9873 obtained for all cases in Figure 8 indicates a high degree of correlation with test data.

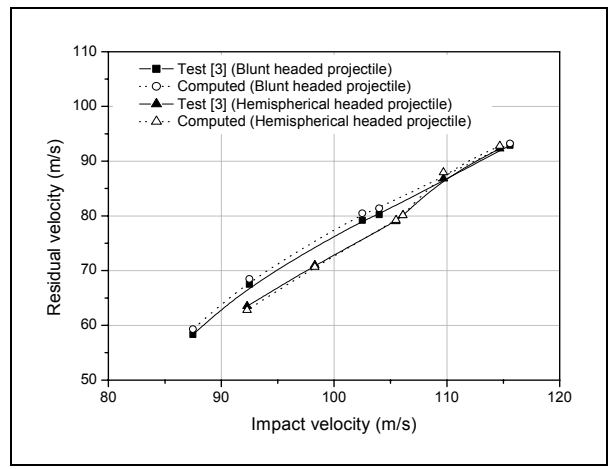

Figure 8: Comparison of computed residual velocities with test results for aluminium plates impacted with blunt and hemispherical projectiles of $19 \mathrm{~mm}$ diameter.

\section{Simulation of target plate perforation}

In laboratory tests of impact on thin aluminium plates, an impinging projectile causes yielding and crack formation in plate material directly on its path leading to perforation, and dishing in the rest of the plate [2]. Views of a typical thin aluminium plate before and after perforation by an ogival-nosed projectile are shown in Figures 9(a) and 9(b) respectively. An analysed plate in Figure 9(c) shows the formation of dishing, and jaggedness along the edge of perforation similar to the 'petalling' phenomenon seen in tests. This can also be observed in the simulation-based snap-shots of plate deformation and failure in course of projectile penetration in Figure 10. A planar view of the analysed plate in Figure 9(d) shows the formation of a circular hole in the plate similar to what was obtained in tests. 


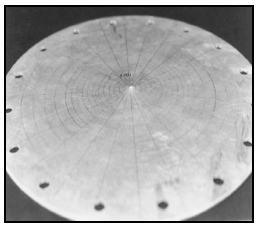

(a)

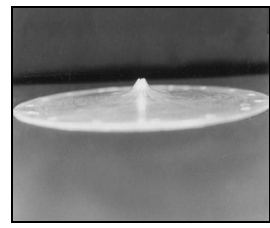

(b)

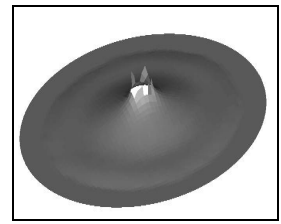

(c)

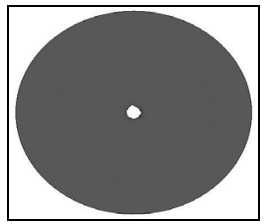

(d)

Figure 9: (a) Plate before impact test [2]; (b) perforated plate after impact test [2]; (c) perforated plate after analysis showing petalling and dishing; (d) planar view of plate after analysis indicating a nearly circular hole.

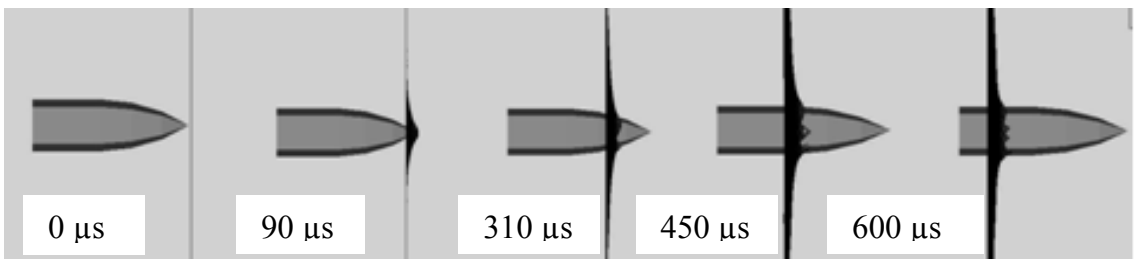

Figure 10: Snap-shots of projectile path during simulation of perforation of a $0.5 \mathrm{~mm}$ thick aluminium plate.

\section{Concluding remarks}

The present paper details a comprehensive numerical study and accurate prediction of ballistic limits of projectiles for normal impact on thin aluminium plates. The material modelling included a relatively straightforward inclusion of strain effects on yield and failure strengths as well as uniaxial failure strain. The elasto-plastic deformations and failure are governed by shear energy criteria for ductile materials as embodied in Von Mises yield criterion together with an associative flow rule of plasticity and isotropic strain hardening. Effects of mesh density and non-inclusion of strain rate effects in constitutive modelling have been studied. It has been shown in a number of cases by comparing with test data that the present modelling and analysis procedure using LS-DYNA is consistently able to predict the effects of plate thickness, projectile mass, and projectile nose shape on ballistic limit and residual velocity. The failure modes obtained through simulation are also realistic when compared to observed plate deformation and perforation in experiments.

\section{References}

[1] Gupta, NK. \& Madhu, V., An experimental study of normal and oblique impact of hard-core projectile on single and layered plates. International Journal of Impact Engineering, 19, pp. 395-414, 1992.

[2] Gupta, NK. Ansari, R. \& Gupta, SK., Normal impact of ogival nosed projectiles on thin plates. International Journal of Impact Engineering, 25, pp. 641-660, 2001. 
[3] Gupta, NK. Iqbal, MA. \& Sekhon, GS. Experimental and numerical studies on the behaviour of thin aluminium plates subjected to impact by blunt- and hemispherical-nosed projectiles. International Journal of Impact Engineering, (Article in press).

[4] Corran, RSJ. Shadbolt, PJ., \& Ruiz, C., Impact loading of plates - An experimental investigation. International Journal of Impact Engineering, 1(1), pp.3-22, 1983.

[5] Backman, ME. \& Goldsmith, W., The mechanics of penetration of projectile into targets. International Journal of Engineering Science, 16, pp.1-99, 1978.

[6] Borvik T., et al., Perforation of $12 \mathrm{~mm}$ thick steel plates by $20 \mathrm{~mm}$ diameter projectiles with flat, hemispherical and conical noses: Part I: Experimental study. International Journal of Impact Engineering, 27, pp.19-35, 2002.

[7] Jones, N., Structural Impact, Cambridge University Press, 1989.

[8] Kad, BK., Schoenfeld, SE. \& Burkins, MS., Through thickness dynamic impact response in textured Ti-6Al-4V plates. Materials Science and Engineering A, 322, pp. 241-251, 2002.

[9] Lim, CT., Shim, VPW. \& Ng, YH., Finite-element modelling of the ballistic impact of fabric armour. International Journal of Impact Engineering, 28, pp. 13-31, 2003.

[10] Tan, VBC. Lim, CT., Cheong, CH., Perforation of high strength fabric by projectiles of different geometry. International Journal of Impact Engineering, 28, pp. 207-222, 2003.

[11] Nandlall, D., Williams, K. \& Vaziri, R., Numerical simulation of the ballistic response of GRP plates. Composites Science and Technology, 58, pp. 1463-1469, 1998.

[12] Park, M., Yoo, J. \& Chung, D-T., An optimisation of multi-layered plate under ballistic impact. International Journal of Solids and Structures, 42, pp.123-137, 2005.

[13] Fawaz, Z., Zheng, W. \& Behdinan. K., Numerical simulation of normal and oblique ballistic impact on ceramic composite armours. Composite Structures 63, pp.387-395, 2004.

[14] Borvik, T. et al., Perforation of $12 \mathrm{~mm}$ thick steel plates by $20 \mathrm{~mm}$ diameter projectiles with flat, hemispherical and conical noses: Part II: Numerical study. International Journal of Impact Engineering, 27, pp.3764, 2002.

[15] Martineau, RL. Prime, MB. \& Duffey, T., Penetration of HSLA-100 steel with tungsten carbide spheres at striking velocities between 0.8 and 2.5 km/s. International Journal of Impact Engineering, 30, pp.505-520, 2004.

[16] www.matweb.com.

[17] Weoi-Shyan L., Jia-Chyuan S. \& Su-Tang C., Effect of strain rate on impact response and dislocation substructure of 6061 T6 aluminium alloy. Scripta Materialia, 42, pp.51-56, 2000. 